\title{
Carcinoma en cuirasse as an initial manifestation of inflammatory breast cancer
}

\author{
Adam Reich ${ }^{1}$, Dominik Samotij ${ }^{1}$, Justyna Szczęch ${ }^{1}$, Zdzisław Woźniak $^{1,2}$, Jacek Szepietowski ${ }^{1}$ \\ 1Department of Dermatology, Venereology and Allergology, Wroclaw Medical University, Wroclaw, Poland
} 2Department of Pathology, Wroclaw Medical University, Wroclaw, Poland

Adv Dermatol Allergol 2016; XXXIII (2): 142-145

DOI: $10.5114 /$ pdia.2015.48069

Carcinoma en cuirasse is an uncommon clinical manifestation of metastatic cutaneous carcinoma characterized by diffuse sclerodermoid induration of the skin. The name was given in the first description by Velpeau in 1838 based on its resemblance to the steel breastplate of cuirassier (cavalry soldier) [1]. Other terms for this distinctive morphological variant of cutaneous metastasis include armoured cancer, Panzerkrebs, scirrhous carcinoma and Acarcine eburnee [2, 3]. Usually carcinoma en cuirasse occurs in patients with local tumour relapse after mastectomy, albeit in some subjects it develops as a presenting feature of primary breast cancer (BC) [4]. Rarely, this form of cutaneous metastasis was associated with other adenocarcinomas (e.g. carcinoma of the lung, kidney or gastrointestinal tract) [5].

A 73-year-old woman, without any relevant concomitant diseases, presented with a 15-month history of asymptomatic, previously untreated erythematous and indurated skin lesions of the right chest. The patient denied any other dermatological conditions, had no family and personal history of malignant neoplasms and did not report such symptoms like fever, night sweats or weight loss.

On admission, the patient was in good general condition, afebrile, with only slight fatigue present. Skin examination disclosed an extensive, prominently indurated shiny infiltration covering the entire range of the markedly retracted right mammary gland extending to the adjacent area of the chest, right arm and upper back (Figure 1). Exceptionally sharp demarcation was particularly striking as the above-described lesion reached the right clavicle with its superior margin spreading down to just reach the inframammary fold with its inferior border. The medial edge of the plaque was distinctly limited by a midsternal line, slightly crossing it and extending laterally to the left parasternal line at the level of the first two ribs. The lateral spread was more linear, extending progressively to the paravertebral line and involving a large part of the right scapular region. The primary infiltration was accompanied by satellite lesions with the most extensive one located in the right epigastric region and the other ones disseminated on the right arm. Notable reduction of the involved breast volume was noted. The nipple, from which no discharge was observed, was neither retracted nor depressed, but could not be invaginated. As a result of induration of the skin and subcutaneous tissue of the affected mammary gland, we could not reliably and thoroughly perform the deep palpation. On further examination, right supraclavicular and axillary lymphadenopathy with subsequent lymphedema of unilateral arm were found. Ultrasound and mammography were performed to exclude any abnormalities in the breast tissue, but both studies were of little help due to a significant degree of skin thickening and the subcutaneous tissue oedema of the right mammary gland and, to a lesser extent, of the left one. Histological examination of the lesional skin biopsy revealed dense, linearly arranged atypical tumour cell infiltrate within the dermis with obstruction of the lymphatic vessels (Figure 2). Immunohistochemical (IHC) staining revealed no expression of oestrogen and progesterone receptors, whereas abundant positive staining of HER2/neu receptors (Figure 2). Providing the presence of histological features typical of both carcinoma en cuirasse and inflammatory breast carcinoma with IHC phenotype specific to the tumours originating from the mammary tissue, the patient was referred to the department of oncology for further treatment-chemotherapy.

Breast cancer is the most common malignancy in women with about $25 \%$ of them developing cutaneous metastases (CMs) in comparison to about 5\% CMs prevalence in the general population of cancer patients [5-7].

Address for correspondence: Adam Reich MD, PhD, Department of Dermatology, Venereology and Allergology, Wroclaw Medical University, 1 Chałubińskiego St, 50-368 Wroclaw, Poland, phone: +48 605076722 , fax: +48 713270999 , e-mail: adam.reich@umed.wroc.pl

Received: 4.10.2014, accepted: 12.11.2014. 

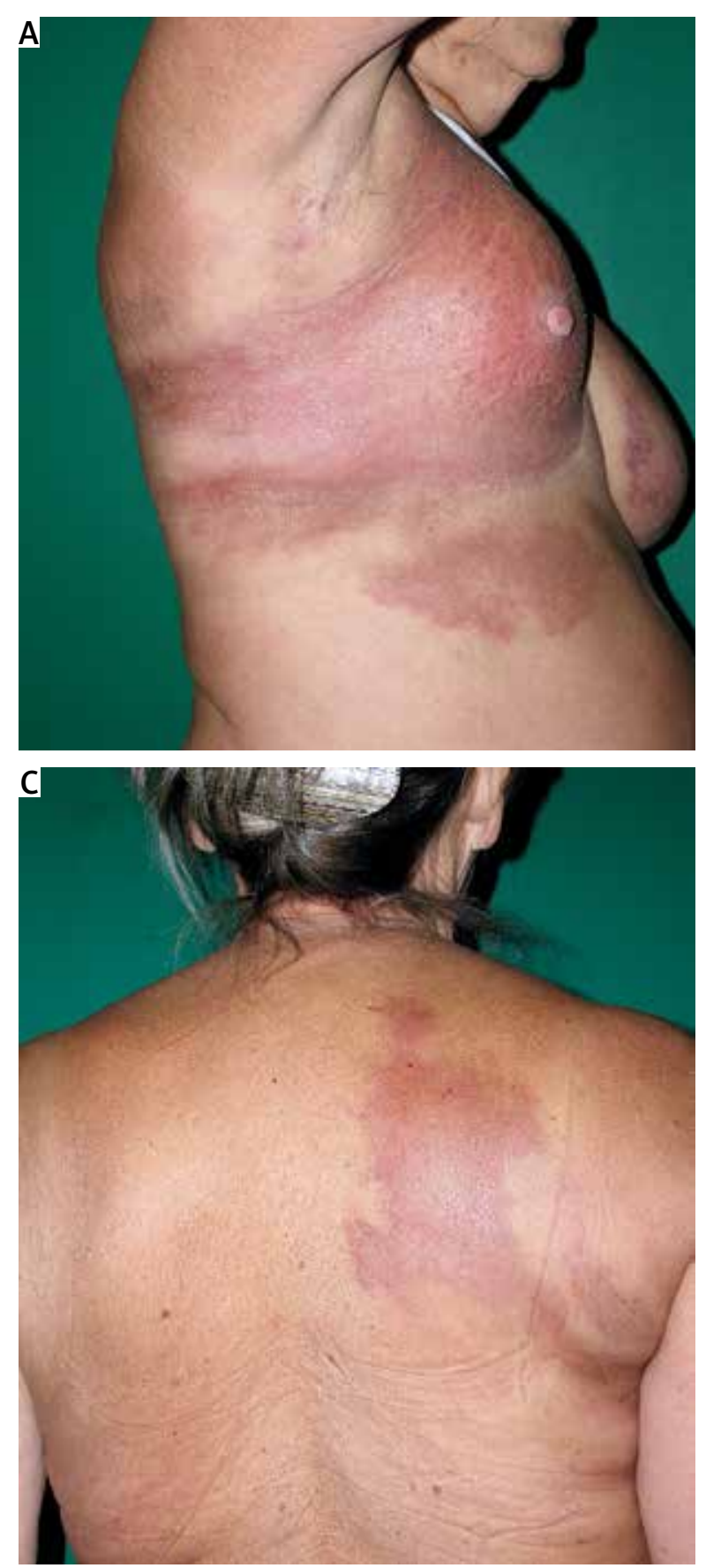

Breast cancer is also the most common source of metastases to skin in females accounting for $69 \%$ of all CMs cases followed by colon cancer, malignant melanoma, and ovarian/cervical cancers [8, 9]. In approximately $12 \%$ of patients the origin of CMs remains unknown [10]. Cutaneous metastases are the initial sign of BC in about $37 \%$ of men and $6 \%$ of women [8]. A recent large metaanalysis revealed that overall frequency of CMs is $5.3 \%$ [6]. The time between diagnosis of $\mathrm{BC}$ and resultant CMs
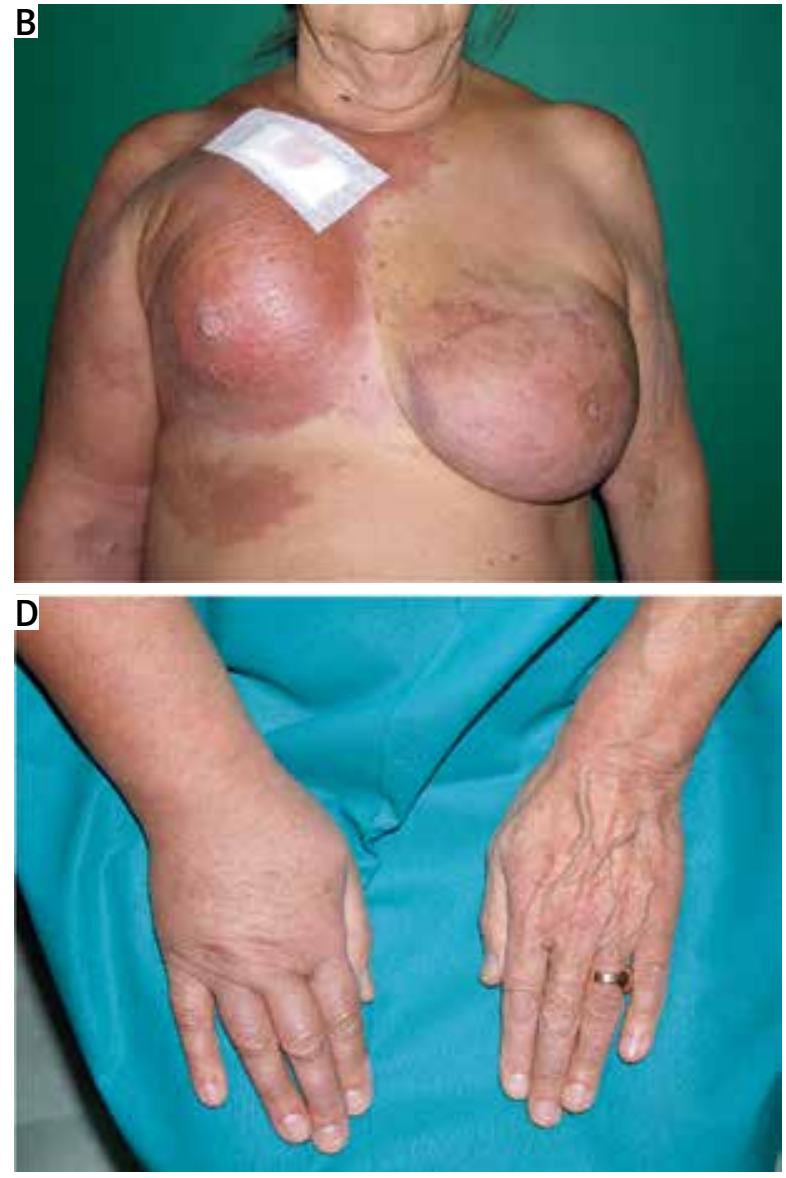

Figure 1. (A) Extensive, prominently indurated skin infiltration covering the entire range of the markedly retracted right mammary gland and (B, C) extending to the adjacent area of the chest, right arm and upper back; (D) lymphedema of the right upper extremity

is variable, although CMs usually occur within the first 3 years after detection of the primary tumour [11]. Metastases to the skin are usually accompanied by internal organ involvement, therefore are associated with an advanced stage of cancer and considered a sign of poor prognosis [7]. Schoenlaub et al. calculated the median survival of patients with CMs from primary BC as 13.8 months in a cohort of 64 patients with only 3.1\% 10-year survival rate [12]. Cutaneous manifestations of BC usually 

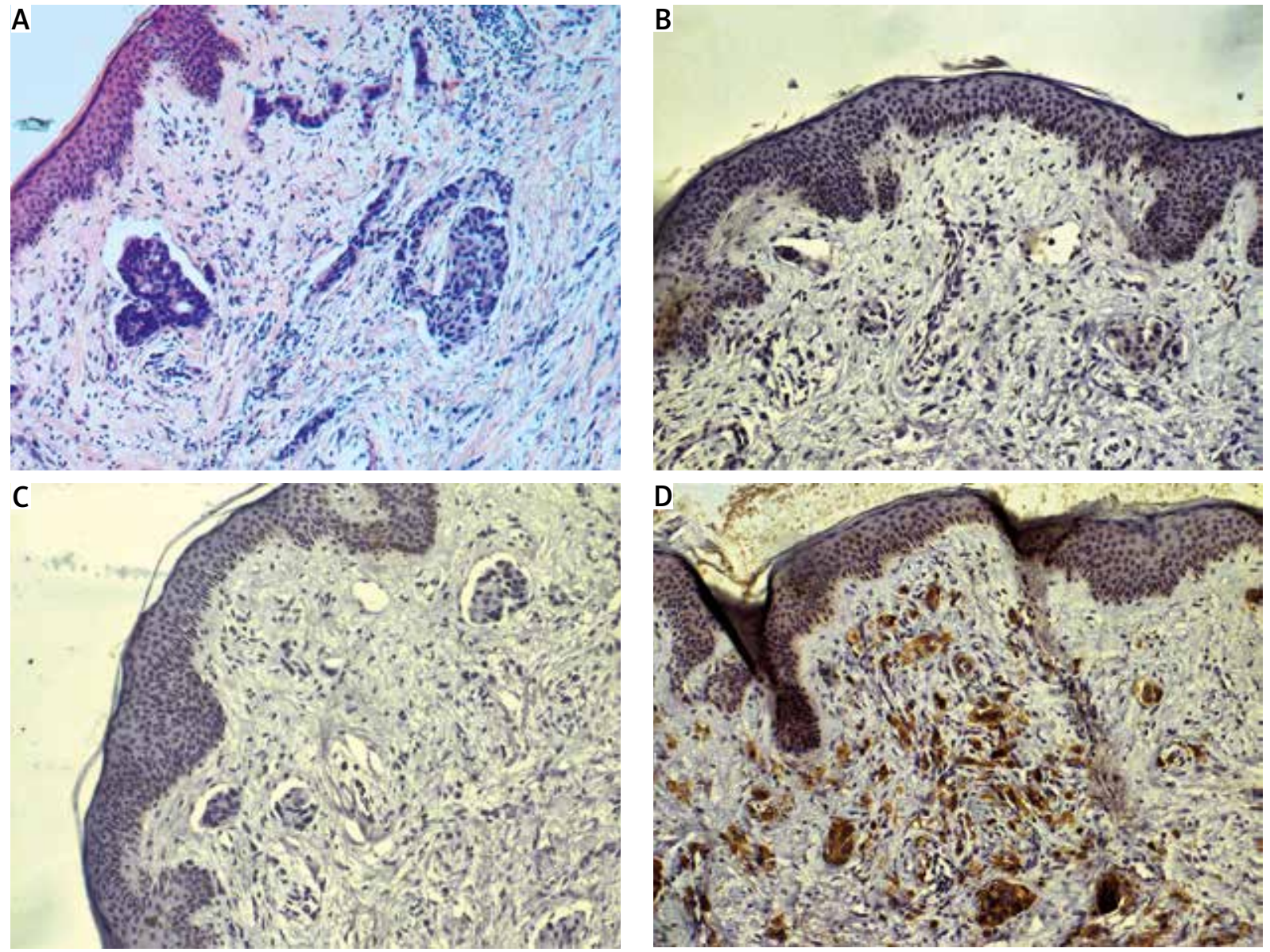

Figure 2. Nests of atypical tumour cells in the dermis (HE, original magnification 100x) (A); tumour cells showed no expression of oestrogen (B) and progesterone receptors (C), but abundant positive staining of HER2/neu receptors (D) (original magnification 100x)

occur in a body region near the primary tumour, most frequently on the anterior aspect of the chest wall, in some cases spreading to the neck, abdomen, axilla and upper back developing from direct extension, blood/lymphatic vessel dissemination, and also surgical implantation with subsequent local occurrence after mastectomy $[5,7]$. Various morphological forms of CMs from BC were described with the most frequent one being solitary to multiple erythematous infiltrating papules and nodules, which accounted for about $80 \%$ of cases according to a large study conducted by Mordenti et al. [4]. The less common, atypical variants of skin involvement in BC can mimic common processes, e.g. erysipelas (carcinoma erysipeloides), alopecia areata (alopecia neoplastica), herpes zoster (zosteriform metastases), lymphangioma circumscriptum, cutaneous vasculitis (carcinoma teleangiectaticum), basal cell carcinoma, squamous cell carcinoma, intertrigo (metastases to the inframammary crest), cutaneous complications from radiotherapy (CMs mimicking radiation dermatitis) and also carcinoma en cuirasse presented here resembling morphea is seen in only $3 \%$ of patients with CMs from BC [4, 5, 13-17]. In those exceptional cases, skin biopsy is often revealing as the histological types of the metastases are usually similar to those of primary tumour, except for anaplastic cases in which IHC markers and ultrastructural examination may be particularly useful [18]. Some authors distinguish eight distinct clinicopathological forms of CMs, including en cuirasse (or so called sclerodermoid carcinoma), histopathologically characterized by interstitial infiltration of tumour cells in single rows or in a cord-arranged, linear ("Indian file") pattern in the dermis with secondary fibrosis of the stroma [19].

Not much data are available on treating BC metastases to the skin, therefore treatment options used in managing all types of BC metastases are preferred. Surgical methods are usually avoided as CMs are frequently a sign of an advanced process. Miltefosine, a topical cytostatic, was employed in the treatment of patients with various forms of CMs with minor results [20]. No particular drug is advised for chemotherapy of CMs exclusively. According to the trial data, capecitabine has shown a re- 
sponse rate ranging from $15 \%$ to $28 \%$ in a population of metastatic breast cancer patients. Sideras et al. [21] have published, however, a report of resolution of skin lesions in 2 patients with cutaneous involvement in the course of BC. Various attempts have also been made to treat this disease with intralesional chemotherapy, radiotherapy, and hormonal antagonists with mixed results [22, 23]. A treatment with combination of the snake venom crotoxin and cardiotoxin called VRCTC-310 used in one study objectively demonstrated a complete response of primary tumour mass with disappearance of supraclavicular tumour mass, as well as a significant reduction of lymphangitis [24]. Recent data show that electrochemotherapy can be an effective and safe loco-regional therapy in patients with CMs, especially in chemotherapy-resistant and radiotherapy-resistant lesions [25].

To the best of our knowledge, very few reports describing the cutaneous involvement representing the initial clinical manifestation of BC have been published to date. In our case, the patient was unaware of the seriousness of the underlying disease and en cuirasse lesions turned out to be her initial complaint. Therefore, we want to underline the primary responsibility of dermatologists, general practitioners and clinicians caring for the breast cancer patients and their ability to recognize certain types of lesions as a possible early sign of unknown internal malignancy. Our report should also prompt clinicians to perform skin biopsy as it is an easily obtainable source of material for histologic verification and other assessments (e.g. IHC, PCR) helpful in establishing the diagnosis and selecting proper treatment modality. Early and accurate recognition only allows to treat the spread of the cancer at the earliest possible stage to improve survival.

\section{Conflict of interest}

The authors declare no conflict of interest.

\section{References}

1. Savatard L. Cancer en cuirasse. Br J Dermatol Syphilis 1943; 55: 31-9.

2. Braun-Falco O, Burgdorf WHC, Plewig G, Wolff HH. Cutaneous metastases. In: Dermatology. Braun-Falco O, Burgdorf WHC, Plewig G, Wolff HH (eds). Springer, Berlin Heidelberg 2000; 1485-87.

3. Carlesimo M, Rossi G, Narcisi A, et al. Carcinoma en cuirasse of the breast. Eur J Dermatol 2009; 19: 289-90.

4. Mordenti C, Peris KM, Concetta Fargnoli M, et al. Cutaneous metastatic breast carcinoma: a study of 164 patients. Acta Dermatoven APA 2000; 9: 143-8.

5. Schwartz RA. Cutaneous metastatic disease. I Am Acad Dermatol 1995; 33: 161-82.

6. Krathen RA, Orengo IF, Rosen T. Cutaneous metastasis: a meta-analysis of data. South Med J 2003; 96: 164-7.

7. Lookingbill DP, Spangler N, Sexton FM. Skin involvement as the presenting sign of internal carcinoma: a retrospective study of 7316 cancer patients. J Am Acad Dermatol 1990; 22: $19-26$

8. Calonje E, Brenn T, Lazar A. Cutaneous metastases and Paget's disease of the skin. In: Pathology of the skin with clinical correlations. McKee PH, Calonje E, Granter SR (eds). Elsevier Mosby, Philadelphia 2005; 1514-8.

9. Brownstein MH, Helwig EB. Patterns of cutaneous metastasis. Arch Dermatol 1972; 105: 862-8.

10. Balzani A, Clerico R, Schwartz RA. Cutaneous implantation metastasis of cholangiocarcinoma after percutaneous transhepatic billiary drainage. Acta Dermatovenerol Croat 2005; 13: 118-21.

11. Johnson WC. Metastatic carcinoma of the skin: incidence and dissemination. In: Lever's histopathology of the skin. Elder D, Elenitsas R, Jaworsky C, Johnson B (eds). Lippincott Williams \& Wilkins, Philadelphia 1997; 1011-8.

12. Schoenlaub P, Sarraux A, Grosshans E, et al. Survival after cutaneous metastasis: a study of 200 cases. Ann Dermatol Venereol 2001; 128: 1310-5.

13. Canpolat F, Akpinar H, Eskioglu F, et al. A case of inflammatory breast carcinoma: carcinoma erysipeloides. Indian J Dermatol Venereol Leprol 2010; 76: 215.

14. Carson HJ, Pellettiere EV, Lack E. Alopecia neoplastica simulating alopecia areata and antedating the detection of primary breast carcinoma. J Cutan Pathol 1994; 21: 67-70.

15. Reich A, Woźniak Z, Szepietowski J. Zosteriform cutaneous metastasis. Dermatol Klin 2006; 8: 49-51.

16. Pickard C, Callen JP, Blumenreich M. Metastatic carcinoma of the breast - an unusual presentation mimicking cutaneous vasculitis. Cancer 1987; 59: 1184-6.

17. Gugle A, Malpathak V, Zarwar V, et al. Carcinoma erysipeloides: an unusual presentation mimicking radiation dermatitis. Dermatol Online J 2008; 14: 26.

18. Saeed S, Keehn CA, Morgan MB. Cutaneous metastasis: a clinical, pathological, and immunohistochemical appraisal. J Cutan Pathol 2004; 3: 419-30.

19. Schwartz RA. Histopathologic aspects of cutaneous metastatic disease. J Am Acad Dermatol 1995; 33: 649-57.

20. Clive S, Gardiner J, Leonard RC. Miltefosine as a topical treatment for cutaneous metastases in breast carcinoma. Cancer Chemother Pharmacol 1999; 44 Suppl.: S29-30.

21. Sideras K, Zahasky KM, Kaur JS. Response of cutaneous metastases from breast cancer to capecitabine. Clin Med Oncol 2008; 2: 415-8.

22. Kumar PP, Good RR, Jones EO, et al. Pathophysiology and rotational subtotal skin electron beam therapy (SSEBT) a new technique. Radiat Med 1989; 7: 95-104.

23. Hehr T, Lamprecht U, Glocker S, et al. Thermoradiotherapy for locally recurrent breast cancer with skin involvement. Int J Hyperthermia 2001; 17: 291-301.

24. Costa LA, Miles H, Araujo CE, et al. Tumor regression of advanced carcinomas following intra- and/or peritumoral inoculation with VRCTC-310 in humans: preliminary report of two cases. Immunopharmacol Immunotoxicol 1998; 20: 15-25.

25. Benevento R, Santoriello A, Perna G, et al. Electrochemotherapy of cutaneous metastasis from breast cancer in elderly patients: a preliminary report. BMC Surgery 2012; 12 (Suppl. 1): S6. 